\title{
The additivity problem in quantum information theory
}

\author{
Alexander S. Holevo*
}

\begin{abstract}
In this lecture we survey the present status of the additivity problem for the classical capacity and related characteristics of quantum channels - one of the most profound mathematical problems of quantum information theory.
\end{abstract}

Mathematics Subject Classification (2000). Primary 94A15; Secondary 81P68.

Keywords. Quantum information theory, quantum channel, classical capacity, entanglement, additivity problem.

\section{Introduction}

The problems of data transmission and storage by quantum information carriers received increasing attention during past decade, owing to the burst of activity in the field of quantum information and computation [42], [22]. At present we are witnessing emergence of theoretical and experimental foundations of the quantum information science. It represents a new exciting research field addressing a number of fundamental issues both in quantum physics and in information and computer sciences. On the other hand, it provides a rich source of well-motivated mathematical problems, often having simple formulations but hard solutions.

A central result in the classical information theory is the coding theorem, establishing the possibility of reliable data transmission and processing at rates lower than the capacity of the communication channel. The issue of the information capacity of quantum channels arose soon after publication of Shannon's pioneering paper and goes back to the works of Gabor, Brillouin and Gordon, asking for fundamental limits on the rate and quality of information transmission. These works laid a physical foundation and raised the question of consistent mathematical treatment of the problem. Important steps in this direction were made in the seventies when quantum statistical decision theory was created, making a noncommutative probability frame for this circle of problems, see [21] for a survey.

A dramatic progress has been achieved during the past decade [42], [6], [22]. In particular, a number of coding theorems was discovered, moreover, it was realized

\footnotetext{
*This work was done partially when the author was Leverhulme Visiting Professor at CQC, DAMTP, University of Cambridge. The work was also supported by the Program "Theoretical Problems of Modern Mathematics" of the Mathematics Division of RAS. The author is grateful to A. Ekert, M. B. Ruskai, M. Shirokov, Yu. M. Suhov and R. F. Werner for fruitful discussions.
} 
that the quantum channel is characterized by the whole spectrum of capacities depending on the nature of the information resources and the specific protocols used for the transmission, see [6], [14]. This new age of quantum information science is characterized by emphasis onto the new possibilities (rather than mere restrictions) inherent in the quantum nature of the information processing agent. On the other hand, the questions of information capacities turned out to be relevant to the theory of quantum computations, particularly in connection with quantum error-correction, communication protocols, algorithmic complexity and a number of other important issues.

The quantum information processing systems have a specifically novel resource, entanglement, a kind of non-classical correlation between parts of the composite quantum system. Among many other unusual features it underlies the strict superadditivity of Shannon information due to entangled decodings in a situation formally similar to the classical memoryless channels [20], [22], [6]. Namely, for independent quantum systems there are entangled measurements which can bear more information than the arithmetic sum of information from these systems. This property has profound consequences for the theory of quantum communication channels and their capacities.

A closely related issue is the additivity of the capacity-related quantities for the memoryless quantum channels with respect to entangled encodings. Should the additivity fail, this would mean that applying entangled inputs to several independent uses of a quantum channel may result in superadditive increase of its capacity for transmission of classical information. However so far there is neither a single evidence of such a non-additivity, nor a general proof for the additivity. In this lecture we survey the present status of this problem.

We start in Section 2 with the classical case, where the additivity holds for almost obvious reasons. We then describe the problem in the finite-dimensional quantum setting in Section 3, discussing also the various formulations of the additivity conjecture and connections between them. Positive results for several concrete classes of channels are briefly surveyed in Section 4, where also an important counterexample to the additivity of the minimal output quantum Rényi entropy is discussed. Since quantum communication channels are described mathematically as completely positive maps, we devote Subsection 4.1 to the description of their structure paying attention to the notion of complementary maps which leads to new examples of additivity. Section 5 is devoted to different formulations of the additivity conjecture using tools from convex analysis. In Subsection 5.4 we present an argument, essentially due to P. Shor, implying the global equivalence of different forms of the additivity conjecture. We conclude with Section 6 , where we briefly outline the works treating the infinite-dimensional case. 


\section{Additivity in the classical information theory}

Let $\mathcal{X}, \mathcal{Y}$ be two finite sets (alphabets), and let $[\Phi(x, y)]_{x \in \mathcal{X}, y \in \mathcal{Y}}$ be a stochastic matrix, i.e.,

1. $\Phi(x, y) \geq 0, x \in \mathcal{X}, y \in \mathcal{Y}$;

2. $\sum_{y \in y} \Phi(x, y)=1, x \in \mathcal{X}$.

In information theory a stochastic matrix describes a (noisy) channel from $\mathcal{X}$ to $\mathcal{Y}$. It transforms an input probability distribution $\pi$ on $\mathcal{X}$ into the output probability distribution $\pi^{\prime}=\Phi \pi$ on $\mathcal{y}$. Denote by

$$
\mathcal{P}(X)=\left\{\pi: \pi(x) \geq 0, \sum_{x \in \mathcal{X}} \pi(x)=1\right\}
$$

the simplex of all probability distributions $\pi$ on $\mathcal{X}$. Extreme points of $\mathcal{P}(\mathcal{X})$ are the degenerate probability distributions $\delta_{x}$ on $\mathcal{X}$. Notice the following obvious property:

For a direct product $\mathcal{X}_{1} \times \mathcal{X}_{2}$ of two alphabets, extreme points of $\mathcal{P}\left(\mathcal{X}_{1} \times \mathcal{X}_{2}\right)$ are precisely the products of extreme points of $\mathcal{P}\left(\mathcal{X}_{j}\right)$ :

$$
\operatorname{ext} \mathcal{P}\left(\mathcal{X}_{1} \times \mathcal{X}_{2}\right)=\operatorname{ext} \mathcal{P}\left(\mathcal{X}_{1}\right) \times \operatorname{ext} \mathcal{P}\left(\mathcal{X}_{2}\right)
$$

The most important characteristic of a channel is its capacity

$$
C(\Phi)=\max _{\pi \in \mathcal{P}(\mathcal{X})}\left\{H(\Phi \pi)-\sum_{x} \pi(x) H\left(\Phi \delta_{x}\right)\right\},
$$

where the expression in curly brackets is equal to the Shannon mutual information between the input and the output of the channel. Here

$$
H(\pi)=-\sum_{x} \pi(x) \log \pi(x)
$$

is the entropy of the probability distribution $\pi$.

One of the main results of information theory - the coding theorem for memoryless channels, see e.g. [10] - says that the quantity (2) is the ultimate rate of asymptotically perfect transmission of information by $n$ independent uses of the channel $\Phi$, when $n \rightarrow \infty$. The capacity has the fundamental additivity property

$$
C\left(\Phi_{1} \otimes \Phi_{2}\right)=C\left(\Phi_{1}\right)+C\left(\Phi_{2}\right) .
$$

Here the inequality $\geq$ (superadditivity) follows by restricting to the independent inputs, while the opposite inequality can be proved by using subadditivity of the output entropy $H(\Phi \pi)$ and the property (1) for the second term in the Shannon information (which is equal to minus the conditional output entropy). The additivity is an important ingredient of the proof of the coding theorem, implying

$$
C\left(\Phi^{\otimes n}\right)=n C(\Phi),
$$


where $\Phi^{\otimes n}=\underbrace{\Phi \otimes \cdots \otimes \Phi}_{n}$. It expresses the "memoryless" character of the information transmission scheme based on the independent uses of the channel. For schemes with memory the capacity can be strictly superadditive.

In what follows we are going to describe the noncommutative analog of the quantity $C(\Phi)$, as well as several other related quantities playing a basic role in quantum information theory. The corresponding additivity property was conjectured to hold also in the noncommutative case, although so far there is neither a general proof, nor a counterexample; moreover the additivity is no longer "natural" since an analog of the underlying basic fact (1) breaks dramatically in the noncommutative case.

\section{Quantum channels}

3.1. The $\chi$-capacity. Let $\mathscr{H}$ be a unitary space and let $\mathfrak{M}(\mathscr{H})$ be the algebra of all linear operators in $\mathscr{H}$. By choosing an orthonormal basis, $\mathscr{H}$ can be identified with the space $\mathscr{H}_{d}$ of $d$-dimensional complex vectors and $\mathfrak{M}(\mathscr{H})$ with the algebra $\mathfrak{M}_{d}$ of complex $d \times d$-matrices.

We shall consider linear maps $\Phi$ which take operators $F$ in $d$-dimensional unitary space $\mathscr{H}$ to operators $F^{\prime}=\Phi(F)$ in a $d^{\prime}$-dimensional space $\mathscr{H}^{\prime}$. Sometimes these are called "superoperators" or "supermatrices" because they can be described as matrices with $d^{2} \times d^{\prime 2}$ entries [9].

Let $\Phi_{j}: \mathfrak{M}\left(\mathscr{H}_{j}\right) \rightarrow \mathfrak{M}\left(\mathcal{H}_{j}^{\prime}\right) ; j=1,2$, be two such maps, and let $\Phi_{1} \otimes \Phi_{2}$ : $\mathfrak{M}\left(\mathscr{H}_{1}\right) \otimes \mathfrak{M}\left(\mathscr{H}_{2}\right) \rightarrow \mathfrak{M}\left(\mathscr{H}_{1}^{\prime}\right) \otimes \mathfrak{M}\left(\mathscr{H}_{2}^{\prime}\right)$ be their tensor product defined by the natural action on product operators and then extended by linearity.

An operator $F \in \mathfrak{M}(\mathscr{H})$ is called positive, $F \geq 0$, if the corresponding matrix is positive semidefinite and the map $\Phi: \mathfrak{M}(\mathscr{H}) \rightarrow \mathfrak{M}\left(\mathscr{H}^{\prime}\right)$ is called positive if $F \geq 0$ implies $\Phi(F) \geq 0$.

Especially important for us will be the class of completely positive (CP) maps [51], [9], [43]. The map $\Phi: \mathfrak{M}(\mathscr{H}) \rightarrow \mathfrak{M}\left(\mathscr{H}^{\prime}\right)$ is completely positive, if for $d=1,2, \ldots$ the maps $\Phi \otimes \operatorname{Id}_{d}$ are all positive, where $\mathrm{Id}_{d}: \mathfrak{M}_{d} \rightarrow \mathfrak{M}_{d}$ is the identity map of the algebra of $d \times d$-matrices. It follows that the tensor product of CP maps is again CP, since

$$
\Phi_{1} \otimes \Phi_{2}=\left(\operatorname{Id}_{d_{1}^{\prime}} \otimes \Phi_{2}\right) \circ\left(\Phi_{1} \otimes \operatorname{Id}_{d_{2}}\right) .
$$

There are positive maps that are not $\mathrm{CP}$, a basic example being provided by the matrix transposition $F \rightarrow F^{\top}$ in a fixed basis.

Finite quantum system is described by a unitary space $\mathscr{H}$. The convex subset

$$
\mathfrak{S}(\mathcal{H})=\{\rho: \rho \geq 0, \operatorname{Tr} \rho=1\}
$$

of $\mathfrak{M}(\mathcal{H})$ is called the quantum state space. The operators $\rho \in \mathfrak{S}(\mathscr{H})$ are called density operators or quantum states. The state space is a compact convex set with the 
extreme boundary

$$
\mathfrak{P}(\mathscr{H})=\operatorname{ext} S(\mathscr{H})=\left\{\rho: \rho \geq 0, \operatorname{Tr} \rho=1, \rho^{2}=\rho\right\} .
$$

Thus extreme points of $\mathfrak{S}(\mathscr{H})$, which are also called pure states, are one-dimensional projectors, $\rho=P_{\psi}$ for a vector $\psi \in \mathscr{H}$ with unit norm, see, e.g. [42], [22].

Instead of the classical relation (1), one has the following relation for a tensor product $\mathscr{H}_{1} \otimes \mathscr{H}_{2}$ of two unitary spaces

$$
\operatorname{ext} S\left(\mathscr{H}_{1} \otimes \mathscr{H}_{2}\right) \supsetneqq \operatorname{ext} S\left(\mathscr{H}_{1}\right) \times \operatorname{extS}\left(\mathscr{H}_{2}\right),
$$

since apparently there are continually many pure states $P_{\psi}$ in $\mathscr{H}_{1} \otimes \mathscr{H}_{2}$, given by vectors $\psi$ not representable as a tensor product $\psi_{1} \otimes \psi_{2}$. In quantum theory the tensor product $\mathscr{H}_{1} \otimes \mathscr{H}_{2}$ describes the composite (bipartite) system. Vectors that are not of the form $\psi_{1} \otimes \psi_{2}$, as well as the corresponding pure states, are called entangled. In an entangled pure state of a bipartite quantum system, neither of the parts is in a pure state, in a sharp contrast to the classical systems.

A CP map $\Phi$ is called a (quantum) channel, if it is trace preserving, i.e. if it maps quantum states into quantum states (possibly in another unitary space $\mathcal{H}^{\prime}$ ). A channel $\Phi$ is called unital if $d=d^{\prime}$ and $\Phi(I)=I^{\prime}$, where $I\left(I^{\prime}\right)$ is the identity operator in $\mathscr{H}$ resp. $\mathscr{H}^{\prime}$.

The von Neumann entropy of a density operator $\rho$

$$
H(\rho)=-\operatorname{Tr} \rho \log \rho
$$

is nonnegative concave continuous function on $\mathfrak{S}(\mathscr{H})$ vanishing on $\mathfrak{P}(\mathcal{H})$ and taking the maximal value $\log d$ on the chaotic state $\rho=\frac{I}{d}$. The noncommutative analog of the quantity (2) is the $\chi$-capacity [20], [22] of the channel $\Phi$,

$$
C_{\chi}(\Phi)=\max _{\pi}\left\{H\left(\Phi\left(\sum_{x} \pi(x) \rho(x)\right)\right)-\sum_{x} \pi(x) H(\Phi(\rho(x)))\right\},
$$

where the maximum is taken over all state ensembles i.e. finite probability distributions $\pi$ on the quantum state space $\mathfrak{S}(\mathscr{H})$ ascribing probabilities $\pi(x)$ to density operators $\rho(x)^{1}$. The additivity conjecture is whether the analog of the property (3) holds for quantum channels, i.e.

$$
C_{\chi}\left(\Phi_{1} \otimes \Phi_{2}\right) \stackrel{?}{=} C_{\chi}\left(\Phi_{1}\right)+C_{\chi}\left(\Phi_{2}\right)
$$

Here again $\otimes$ is the tensor product of the two channels describing independent uses of the channels on the states of the composite system. This is the earliest additivity conjecture in quantum information theory which can be traced back to [5], see also [20], [6].

\footnotetext{
${ }^{1}$ In the finite dimensional case we are considering the maximum is indeed attained on $\pi$ with support having at most $d^{2}$ states, where $d=\operatorname{dim} \mathscr{H}[50]$.
} 
In physical terms this problem can also be formulated as: "Can entanglement between input states help to send classical information through quantum channels?" The classical capacity of a quantum channel is defined as the maximal transmission rate per use of the channel, with coding and decoding chosen for an increasing number $n$ of independent uses of the channel

$$
\Phi^{\otimes n}=\underbrace{\Phi \otimes \cdots \otimes \Phi}_{n}
$$

such that the error probability goes to zero as $n \rightarrow \infty$. A basic result of quantum information theory - the quantum coding theorem [19], [49]-implies that the classical capacity $C(\Phi)$ and the $\chi$-capacity $C_{\chi}(\Phi)$ are connected by the formula

$$
C(\Phi)=\lim _{n \rightarrow \infty}(1 / n) C_{\chi}\left(\Phi^{\otimes n}\right) .
$$

Since $C_{\chi}(\Phi)$ is easily seen to be superadditive, i.e.

$$
C_{\chi}\left(\Phi_{1} \otimes \Phi_{2}\right) \geq C_{\chi}\left(\Phi_{1}\right)+C_{\chi}\left(\Phi_{2}\right)
$$

one has $C(\Phi) \geq C_{\chi}(\Phi)$. If the additivity (6) holds, then $C_{\chi}\left(\Phi^{\otimes n}\right)=n C_{\chi}(\Phi)$, and this would imply $C(\Phi)=C_{\chi}(\Phi)$. Such a result would be very much welcome from a mathematical point of view, giving a relatively easily computable "single-letter" expression for the classical capacity of a quantum channel.

On the other hand, such an equality is rather counter-intuitive in view of the relation (4) and existence of waste variety of pure entangled states. In fact, there are several quantities that are nonadditive under the tensor product of quantum channels such as: a) the Shannon information maximized over entangled outputs [20]; b) the quantum capacity [6]; c) the minimal output Rényi entropy [53] and some others, the classical counterparts of which are additive. In the following we shall consider the case c) which is most relevant to our main problem (6).

3.2. Entropic characteristics of $\mathbf{C P}$ maps and channels. The quantum Rényi entropy of order $p>1$ of a density operator $\rho$ is defined as

$$
R_{p}(\rho)=\frac{1}{1-p} \log \operatorname{Tr} \rho^{p},
$$

so that the minimal output Rényi entropy of the channel $\Phi$ is

$$
\check{R}_{p}(\Phi)=\min _{\rho \in \mathfrak{S}(\mathcal{H})} R_{p}(\Phi(\rho))=\frac{p}{1-p} \log v_{p}(\Phi),
$$

where

$$
v_{p}(\Phi)=\max _{\rho \in \mathfrak{S}(\mathscr{H})}\left[\operatorname{Tr} \Phi(\rho)^{p}\right]^{1 / p}
$$


is a "measure of output purity" of the channel $\Phi$ introduced in [3] $]^{2}$. In the limit $p \downarrow 1$ the quantum Rényi entropies monotonically increase and uniformly converge to the entropy of a density operator $\rho$,

$$
\lim _{p \downarrow 1} R_{p}(\rho)=H(\rho),
$$

so that introducing the minimal output entropy

$$
\check{H}(\Phi)=\min _{\rho \in \mathfrak{S}(\mathscr{H})} H(\Phi(\rho))
$$

of the quantum channel $\Phi$, one has $\lim _{p \downarrow 1} \check{R}_{p}(\Phi)=\check{H}(\Phi)$.

The classical analog of the quantity $(8)$ is

$$
v_{p}(\Phi)=\max _{\pi \in \mathcal{P}(X)}\|\Phi \pi\|_{p}
$$

where $\|f\|_{p}=\left(\sum_{x \in \mathcal{X}}|f(x)|^{p}\right)^{1 / p}$ is the $l_{p}$-norm of $f=(f(x))_{x \in \mathcal{X}}$. The function $\pi \rightarrow\|\Phi \pi\|_{p}$ is convex continuous and hence attains the maximum at an extreme point of $\mathcal{P}(\mathcal{X})$, i.e. on a degenerate probability distribution $\delta_{x}$. Hence the basic property (1) implies the multiplicativity relation

$$
v_{p}\left(\Phi_{1} \otimes \Phi_{2}\right)=v_{p}\left(\Phi_{1}\right) v_{p}\left(\Phi_{2}\right),
$$

which is equivalent to the additivity property of the minimal output Rényi entropies

$$
\check{R}_{p}\left(\Phi_{1} \otimes \Phi_{2}\right)=\check{R}_{p}\left(\Phi_{1}\right)+\check{R}_{p}\left(\Phi_{2}\right)
$$

implying in turn

$$
\check{H}\left(\Phi_{1} \otimes \Phi_{2}\right)=\check{H}\left(\Phi_{1}\right)+\check{H}\left(\Phi_{2}\right),
$$

in the limit $p \downarrow 1$. Notice that the inequality $\leq$ is obvious in (11), (12).

Unlike the classical case, there is no apparent reason for these multiplicativity/additivity properties to hold in the case of quantum channels. Nevertheless there are several important classes of channels for which the multiplicativity (10) can be proved for all $p>1$, although there is also an example where it breaks for sufficiently large $p$. This, however, does not preclude that it can hold for $p$ close to 1 , and the validity of $(10)$ for $p \in(1,1+\varepsilon)$, with $\varepsilon>0$, implies validity of the additivity property (12), which, as we shall see, is closely related to the additivity of the $\chi$-capacity (6).

Here we would also like to mention that multiplicativity of more general $(q \rightarrow p)$ norms was studied for the cases where at least some of the maps $\Phi_{1}, \Phi_{2}$ is not CP, see [38], [37], [35]. Basing on the advanced theory of the operator $L_{p}$-spaces [44], [43], there is an interesting study concerning the multiplicativity of completely bounded $p$-norms, which however is related to the additivity of a completely different entropic quantity [15].

\footnotetext{
${ }^{2}$ In the finite-dimensional case all the functions of the state we are considering are easily seen to be continuous and their extrema on the state space are attained. However it is not so in infinite-dimensional case, and then the attainability of the extrema requires separate study, see Section 6.
} 


\section{Some classes of CP maps and channels}

4.1. Representations of $\mathbf{C P}$ maps. Here we recollect some facts concerning the structure of CP maps and channels. Given three unitary spaces $\mathscr{H}_{A}, \mathscr{H}_{B}, \mathscr{H}_{C}$ and a linear operator $V: \mathscr{H}_{A} \rightarrow \mathscr{H}_{B} \otimes \mathscr{H}_{C}$, the relation

$$
\Phi(\rho)=\operatorname{Tr}_{\mathscr{H}_{C}} V \rho V^{*}, \quad \tilde{\Phi}(\rho)=\operatorname{Tr}_{\mathscr{H}_{B}} V \rho V^{*} ; \quad \rho \in \mathfrak{M}\left(\mathscr{H}_{A}\right)
$$

defines two CP maps $\Phi: \mathfrak{M}\left(\mathscr{H}_{A}\right) \rightarrow \mathfrak{M}\left(\mathscr{H}_{B}\right), \tilde{\Phi}: \mathfrak{M}\left(\mathscr{H}_{A}\right) \rightarrow \mathfrak{M}\left(\mathscr{H}_{C}\right)$, which are called mutually complementary [25] (or conjugate [36]). If $V$ is an isometry then both maps are channels.

For any linear map $\Phi: \mathfrak{M}(\mathscr{H}) \rightarrow \mathfrak{M}\left(\mathscr{H}^{\prime}\right)$ the dual map $\Phi^{*}: \mathfrak{M}\left(\mathscr{H}^{\prime}\right) \rightarrow \mathfrak{M}(\mathscr{H})$ is defined by the formula

$$
\operatorname{Tr} \Phi(\rho) X=\operatorname{Tr} \rho \Phi^{*}(X) ; \quad \rho \in \mathfrak{M}(\mathscr{H}), \quad X \in \mathfrak{M}\left(\mathscr{H}^{\prime}\right) .
$$

If $\Phi$ is CP, then $\Phi^{*}$ is also CP. The relations (13) are equivalent to

$$
\begin{array}{ll}
\Phi^{*}(X)=V^{*}\left(X \otimes I_{C}\right) V ; & X \in \mathfrak{M}\left(\mathscr{H}_{B}\right), \\
\tilde{\Phi}^{*}(X)=V^{*}\left(I_{B} \otimes X\right) V ; & X \in \mathfrak{M}\left(\mathscr{H}_{C}\right) .
\end{array}
$$

The Stinespring dilation theorem [51] concerning CP maps on arbitrary $C^{*}$-algebras, for the particular case in question amounts to the statement that for a given $\mathrm{CP}$ map there are a space $\mathscr{H}_{C}$ and an operator $V$ satisfying (14). This implies that given a CP map $\Phi$, a complementary map $\tilde{\Phi}$ always exists.

By introducing a basis $\left\{e_{j}^{C}\right\}$ in $\mathscr{H}_{C}$ and operators $V_{j}: \mathscr{H}_{A} \rightarrow \mathscr{H}_{B}$ defined by

$$
\left(\varphi, V_{j} \psi\right)=\left(\varphi \otimes e_{j}^{C}, V \psi\right) ; \quad \varphi \in \mathscr{H}_{B}, \psi \in \mathscr{H}_{A},
$$

the first relation in (13) can be rewritten as

$$
\Phi(\rho)=\sum_{j=1}^{d_{C}} V_{j} \rho V_{j}^{*} ; \quad \rho \in \mathfrak{M}\left(\mathcal{H}_{A}\right) .
$$

The map (16) is a channel if and only if $\sum_{j=1}^{d_{C}} V_{j}^{*} V_{j}=I$. The relation (16) is usually called the Kraus representation (see also Choi [9]). Of course, there are similar representations for the complementary map $\tilde{\Phi}$ and the dual maps.

Theorem 4.1 ([25], [36]). If one of the relations (11), (12) holds for the CP maps (channels) $\Phi_{1}, \Phi_{2}$, then similar relations holds for the pair of their complementary maps $\tilde{\Phi}_{1}, \tilde{\Phi}_{2}$. If one of these relations holds for a given $\Phi_{1}$ and arbitrary $\Phi_{2}$, then a similar relation holds for the complementary map $\tilde{\Phi}_{1}$ and arbitrary $\Phi_{2}$.

Validity of the multiplicativity conjecture (10) for all $p \geq 1$ and of the additivity conjectures (12), (6) was established in a number of cases where one channel is arbitrary and the other belongs to one of the classes we are going to discuss. 
4.2. Entanglement-breaking maps and their complementary maps. Any linear map $\Phi: \mathfrak{M}(\mathscr{H}) \rightarrow \mathfrak{M}\left(\mathscr{H}^{\prime}\right)$ admits a representation

$$
\Phi(X)=\sum_{j} N_{j}^{\prime} \operatorname{Tr} X M_{j}
$$

where $\left\{M_{j}\right\},\left\{N_{j}^{\prime}\right\}$ are finite collections of operators in $\mathscr{H}$ and $\mathscr{H}^{\prime}$, respectively. This simply follows from the finite dimensionality of $\mathscr{H}, \mathscr{H}^{\prime}$ and the fact that any linear functional on $\mathfrak{M}(\mathscr{H})$ has the form $X \rightarrow \operatorname{Tr} X M$, where $M \in \mathfrak{M}(\mathcal{H})$.

Proposition 4.2. For a linear map $\Phi: \mathfrak{M}(\mathscr{H}) \rightarrow \mathfrak{M}\left(\mathscr{H}^{\prime}\right)$ the following conditions are equivalent:

(i) There is a representation (17) such that $M_{j} \geq 0, N_{j}^{\prime} \geq 0$.

(ii) The map $\Phi$ is $C P$ and has the representation (16) with rank one operators $V_{j}$.

(iii) For $d=2,3, \ldots$ and any $\rho_{12} \in \mathfrak{S}\left(\mathscr{H} \otimes \mathscr{H}_{d}\right)$,

$$
\left(\Phi \otimes \operatorname{Id}_{d}\right)\left(\rho_{12}\right)=\sum_{\alpha} A_{\alpha} \otimes B_{\alpha}
$$

where $A_{\alpha} \geq 0$ and $B_{\alpha} \geq 0$.

Channels satisfying the condition (i) were introduced in [20], and the above characterization was obtained in [29] where such maps were termed entanglement-breaking. In the case of channels, (18) means that the output state $\left(\Phi \otimes \operatorname{Id}_{d}\right)\left(\rho_{12}\right)$ is always separable, i.e. a convex combination of (unentangled) product states. Entanglementbreaking channels can be written in the form

$$
\Phi(\rho)=\sum_{j} \rho_{j}^{\prime} \operatorname{Tr} \rho M_{j},
$$

where $\left\{\rho_{j}^{\prime}\right\}$ is a finite collection of states in $\mathscr{H}^{\prime}$, and $\left\{M_{j}\right\}$ a resolution of the identity in $\mathscr{H}$, i.e. a collection of operators satisfying

$$
M_{j} \geq 0, \quad \sum_{j} M_{j}=I .
$$

Resolutions of the identity describe quantum observables [22], and the channel (19) corresponds to a measurement of the observable $\left\{M_{j}\right\}$ over an input state $\rho$ resulting in a probability distribution $\left\{\operatorname{Tr} \rho M_{j}\right\}$, which is followed by preparation of the output state $\rho_{j}^{\prime}$. Thus, there is a classical information processing stage inside the channel which is responsible for the entanglement-breaking.

The simplest example is the completely depolarizing channel

$$
\Phi(\rho)=\frac{I}{d} \operatorname{Tr} \rho
$$

which maps an arbitrary state to the chaotic state $\frac{I}{d}$. 
As shown in [25], [36], the complementary maps to the entanglement-breaking maps have the form

$$
\tilde{\Phi}(\rho)=\sum_{j, k=1}^{d_{C}} c_{j k}\left\langle\psi_{j}|\rho| \psi_{k}\right\rangle E_{j k}, \quad \rho \in \mathfrak{M}\left(\mathscr{H}_{A}\right),
$$

where $\left[c_{j k}\right]$ is a nonnegative definite matrix, $\left\{\psi_{j}\right\}_{j=\overline{1, d_{C}}}$ a system of vectors in $\mathscr{H}_{A}$, and the $E_{j k}$ 's are the matrix units in $\mathscr{H}_{C}$. In the special case where $\left\{\psi_{j}\right\}_{j=\overline{1, d_{C}}}$ is an orthonormal basis, (20) is the diagonal CP map in the sense of [31]. Diagonal channels are characterized by the additional property $c_{j j} \equiv 1$.

A simplest example of the diagonal channel is the ideal channel Id, which is complementary to the completely depolarizing channel.

For general entanglement-breaking channels the additivity property (12) with arbitrary second channel was established by Shor [47], preceded by results in [20] on special subclasses of such channels. The multiplicativity property (10) for all $p>1$ was established by King [32], basing on the Lieb-Thirring inequality [40]: for $A, B \in \mathfrak{M}(\mathcal{H}), A, B \geq 0$, and $p \geq 1$

$$
\operatorname{Tr}(A B)^{p} \leq \operatorname{Tr} A^{p} B^{p} .
$$

By Theorem 4.1 this implies the corresponding properties for the complementary maps and channels of the form (20).

4.3. Covariant channels. Let $G$ be a group (either finite or continuous) and let $g \rightarrow U_{g}^{A}, U_{g}^{B}, g \in G$, be two projective (unitary) representations of $G$ in $\mathscr{H}_{A}, \mathscr{H}_{B}$. The CP map $\Phi: \mathfrak{M}\left(\mathscr{H}_{A}\right) \rightarrow \mathfrak{M}\left(\mathscr{H}_{B}\right)$ is covariant if

$$
\Phi\left(U_{g}^{A} \rho U_{g}^{A *}\right)=U_{g}^{B} \Phi(\rho) U_{g}^{B *}
$$

for all $g \in G$ and all $\rho$. For a covariant CP map there exists a covariant Stinespring dilation: namely, there is a projective representation $g \rightarrow U_{g}^{C}$ in $\mathscr{H}_{B}$, such that

$$
\left(U_{g}^{B} \otimes U_{g}^{C}\right) V=V U_{g}^{A},
$$

see e.g. [24]. It follows that the complementary map is also covariant:

$$
\tilde{\Phi}\left(U_{g}^{A} \rho U_{g}^{A *}\right)=U_{g}^{C} \tilde{\Phi}(\rho) U_{g}^{C *} .
$$

Lemma 4.3. If the representation $U_{g}^{A}$ is irreducible, then

$$
C_{\chi}(\Phi)=H\left(\Phi\left(\frac{I_{A}}{d_{A}}\right)\right)-\check{H}(\Phi) .
$$

Since the tensor product of irreducible representations of possibly different groups $G_{1}, G_{2}$ is an irreducible representation of the group $G_{1} \times G_{2}$, it follows that the additivity properties (12) and (6) are equivalent for channels satisfying the condition of Lemma 4.3. Symmetry considerations also help to compute explicitly the entropic characteristics of covariant channels. Then, in the case of additivity, $C=C_{\chi}$ gives an explicit expression for the classical capacity of the channel. 
4.4. The unital qubit channels. The simplest and yet fundamental quantum system is the qubit (quantum bit), where $\operatorname{dim} \mathscr{H}=2$. A convenient basis in $\mathfrak{M}_{2}$ is formed by the Pauli matrices

$$
\sigma_{0} \equiv I=\left[\begin{array}{ll}
1 & 0 \\
0 & 1
\end{array}\right], \quad \sigma_{x}=\left[\begin{array}{ll}
0 & 1 \\
1 & 0
\end{array}\right], \quad \sigma_{y}=\left[\begin{array}{cc}
0 & -i \\
i & 0
\end{array}\right], \quad \sigma_{z}=\left[\begin{array}{cc}
1 & 0 \\
0 & -1
\end{array}\right] .
$$

It is known [45] that an arbitrary unital channel $\Phi: \mathfrak{M}_{2} \rightarrow \mathfrak{M}_{2}$ can be decomposed as

$$
\Phi(\rho)=U_{2} \Lambda\left(U_{1} \rho U_{1}^{*}\right) U_{2}^{*},
$$

where $U_{1}, U_{2}$ are unitary matrices and $\Lambda$ has the following canonical Kraus representation:

$$
\Lambda(\rho)=\sum_{\gamma=0, x, y, z} \mu_{\gamma} \sigma_{\gamma} \rho \sigma_{\gamma}
$$

where $\left\{\mu_{\gamma}\right\}$ is a probability distribution. The unital qubit channels (25) are covariant with respect to the projective representation of the group $\mathbb{Z}_{2} \times \mathbb{Z}_{2}$ defined by

$$
U_{00}=\sigma_{0}, \quad U_{01}=\sigma_{z}, \quad U_{10}=\sigma_{x}, \quad U_{11}=-i \sigma_{y} .
$$

Therefore the relation (23) holds for this class of channels.

By using a convex decomposition into diagonal channels of special form and applying to these the Lieb-Thirring inequality (21), King [33] established (10) for all $p>1,(12)$ and (6) for the case where $\Phi_{1}$ is an arbitrary unital qubit channel and $\Phi_{2}$ is an arbitrary channel. There are recent positive results concerning nonunital qubit channels [35].

4.5. Depolarizing channel. The depolarizing channel in $\mathscr{H}_{d}$ is

$$
\Phi(\rho)=(1-p) \rho+p \frac{I}{d} \operatorname{Tr} \rho, \quad 0 \leq p \leq \frac{d^{2}}{d^{2}-1} .
$$

If $p \leq 1$ this describes a mixture of the ideal channel Id and the completely depolarizing channel. For the whole range $0 \leq p \leq \frac{d^{2}}{d^{2}-1}$ complete positivity can be proven by using the Kraus decomposition, see e.g. [42]. The depolarizing channel is characterized by the property of unitary covariance,

$$
\Phi\left(U \rho U^{*}\right)=U \Phi(\rho) U^{*}
$$

for an arbitrary unitary operator $U$ in $\mathcal{H}$.

The properties (10) for all $p>1,(12)$ and (6) were proved in [34] for the case where $\Phi_{1}$ is a depolarizing channel and $\Phi_{2}$ is arbitrary, using a method similar to the case of the unital qubit channels.

Complementarity for depolarizing channels is computed in [11]. 
4.6. A transpose-depolarizing channel. Let us consider in some detail the extreme transpose-depolarizing channel

$$
\Phi(\rho)=\frac{1}{d-1}\left[I \operatorname{Tr} \rho-\rho^{\top}\right]
$$

where $\rho^{\top}$ is the transpose of $\rho$ in an orthonormal basis $\left\{e_{j}\right\}$ in $\mathscr{H}_{d}$. Complete positivity of the map (27) follows from the representation

$$
\Phi(\rho)=\frac{1}{2(d-1)} \sum_{j, k=1}^{d}\left(E_{j k}-E_{k j}\right) \rho\left(E_{j k}-E_{k j}\right)^{*} .
$$

It has the covariance property

$$
\Phi\left(U \rho U^{*}\right)=\bar{U} \Phi(\rho) \bar{U}^{*}
$$

for an arbitrary unitary $U$, where $\bar{U}$ is complex conjugate in the basis $\left\{e_{j}\right\}$. It follows that the relation (23) holds for this channel.

This channel is interesting in that it breaks the additivity of the minimal Rényi entropy (11) with $\Phi_{1}=\Phi_{2}=\Phi$ for $d>3$ and large enough $p$ [53]. At the same time it fulfills (12), see [41], [12], and even (11) for $1 \leq p \leq 2$ [13]. For generalizations to broader classes of channels as well as to the more general forms of additivity, see [41], [1], [54]. This example also shows that although the Lieb-Thirring inequality can be used in several cases to prove the additivity conjecture (11) for all $p>1$, it cannot serve for a general proof. Moreover, there is even no general proof covering all these cases, since each time application of the Lieb-Thirring inequality is supplied with an argument specific to the case under consideration.

The complementary channel which shares the multiplicativity/additivity properties with the channel (27) is

$$
\tilde{\Phi}(\rho)=\frac{2}{(d-1)} P_{-}\left(\rho \otimes I_{2}\right) P_{-}
$$

(see [25] for more details). Here $P_{-}$is the projector onto the antisymmetric subspace of $\mathscr{H} \otimes \mathscr{H}$ of dimension $\frac{d(d-1)}{2}$. The covariance property of the channel (29) is

$$
\tilde{\Phi}\left(U \rho U^{*}\right)=(U \otimes U) \tilde{\Phi}(\rho)\left(U^{*} \otimes U^{*}\right),
$$

as follows from the fact that $P_{-}(U \otimes U)=(U \otimes U) P_{-}$.

\section{A hierarchy of the additivity conjectures}

5.1. Convex closure. To find out the intrinsic connection between the output entropy and the $\chi$-capacity, let us define the average $\bar{\rho}_{\pi}=\sum_{x} \pi(x) \rho(x)$ of the ensemble $\pi$ and rewrite the expression (5) in the form

$$
C_{\chi}(\Phi)=\max _{\rho \in \mathfrak{S}(\mathcal{H})}\left[H(\Phi(\rho))-\hat{H}_{\Phi}(\rho)\right]
$$


where

$$
\hat{H}_{\Phi}(\rho)=\min _{\pi: \bar{\rho}_{\pi}=\rho} \sum_{x} \pi(x) H(\Phi(\rho(x)))
$$

is the convex closure [30] of the output entropy $H(\Phi(\rho))^{3}$. The function $\hat{H}_{\Phi}(\rho)$ is a natural generalization of another important quantity in quantum information theory, namely the "entanglement of formation" [6] and reduces to it when the channel $\Phi$ is a partial trace. This quantity has the conjectured superadditivity property: for an arbitrary state $\rho_{12} \in \mathfrak{S}\left(\mathscr{H}_{1} \otimes \mathscr{H}_{2}\right)$ and arbitrary channels $\Phi_{1}, \Phi_{2}$,

$$
\hat{H}_{\Phi_{1} \otimes \Phi_{2}}\left(\rho_{12}\right) \geq \hat{H}_{\Phi_{1}}\left(\rho_{1}\right)+\hat{H}_{\Phi_{2}}\left(\rho_{2}\right),
$$

where $\rho_{1}, \rho_{2}$ are the partial traces of $\rho_{12}$ in $\mathscr{H}_{1}, \mathscr{H}_{2}$.

It is not difficult to see that this property implies additivity of both the minimal output entropy and the $\chi$-capacity:

Proposition 5.1. The superadditivity property (31) implies the additivity properties (12) and (6) for given channels $\Phi_{1}, \Phi_{2}$.

In the spirit of Theorem 4.1, one can prove ([25]) that

if the relation (31) holds for the pair of CP maps (channels) $\Phi_{1}, \Phi_{2}$, then similar relation holds for the pair of their complementary maps $\tilde{\Phi}_{1}, \tilde{\Phi}_{2}$. If one of these relations holds for given $\Phi_{1}$ and arbitrary $\Phi_{2}$, then a similar relation holds for the complementary map $\tilde{\Phi}_{1}$ and arbitrary $\Phi_{2}$.

Let $\left\{p_{j}\right\}$ be a finite probability distribution and let $\Phi_{j}: \mathfrak{M}(\mathcal{H}) \rightarrow \mathfrak{M}\left(\mathscr{H}_{j}^{\prime}\right)$ be a collection of channels. The channel $\Phi: \mathfrak{M}(\mathscr{H}) \rightarrow \mathfrak{M}\left(\sum_{j} \oplus \mathscr{H}_{j}^{\prime}\right)$ is called orthogonal convex sum of the channels $\Phi_{j}$, if $\Phi(\rho)=\sum_{j} \oplus p_{j} \Phi_{j}(\rho)$ for all $\rho \in \mathfrak{S}(\mathscr{H})$.

Proposition 5.2 ([26]). Let $\Phi_{2}$ be an arbitrary channel. The properties (11), (12), (31) hold if $\Phi_{1}$ is an orthogonal convex sum of either an ideal channel or completely depolarizing channel and a channel $\Phi^{(0)}$ such that the corresponding property holds for $\Phi^{(0)}$ and $\Phi_{2}$.

It follows that such a $\Phi_{1}$ fulfils the additivity of $\chi$-capacity (6).

In this way, for example, one obtains all the additivity properties for the important case of the erasure channel

$$
\Phi(\rho)=\left[\begin{array}{cc}
p \rho & 0 \\
0 & (1-p) \operatorname{Tr} \rho
\end{array}\right]
$$

as it is the orthogonal convex sum of an ideal and a completely depolarizing channel.

\footnotetext{
${ }^{3}$ Here the same comment applies as to the attainability of the maximum in (5).
} 
5.2. Additivity for constrained channels. In this section we consider several equivalent formulations of the additivity conjecture for channels with arbitrarily constrained inputs [26], which formally is substantially stronger than additivity of the unconstrained $\chi$-capacity. Let us denote

$$
\chi_{\Phi}(\rho)=H(\Phi(\rho))-\hat{H}_{\Phi}(\rho),
$$

then the function $\chi_{\Phi}(\rho)$ is continuous and concave on the set $\mathfrak{S}(\mathscr{H})$ of all states in $\mathscr{H}$.

Consider the constraint on the ensemble $\pi$ with the average $\bar{\rho}_{\pi}=\sum_{x} \pi(x) \rho(x)$, defined by the requirement $\bar{\rho}_{\pi} \in \mathcal{A}$, where $\mathcal{A}$ is a closed subset of states. A particular case is the linear constraint $\mathcal{A}=\{\rho: \operatorname{Tr} \rho A \leq \alpha\}$ for a positive operator $A$ and a number $\alpha \geq 0$. Define the $\chi$-capacity of the A-constrained channel $\Phi$ by

$$
C_{\chi}(\Phi ; \mathcal{A})=\max _{\rho \in \mathcal{A}} \chi_{\Phi}(\rho)
$$

Note that the $\chi$-capacity for the unconstrained channel is $C_{\chi}(\Phi)=C(\Phi ; \mathfrak{S}(\mathscr{H}))$. On the other hand, $\chi_{\Phi}(\rho)=C_{\chi}(\Phi ;\{\rho\})$.

Let $\Phi_{1}, \Phi_{2}$ be two channels with the constraints $\mathcal{A}_{1}, \mathcal{A}_{2}$. For the channel $\Phi_{1} \otimes \Phi_{2}$ we introduce the constraint $\mathscr{A}_{1} \otimes \mathcal{A}_{2} \equiv\left\{\rho: \operatorname{Tr}_{\mathcal{H}_{2}} \rho \in \mathcal{A}_{1}, \operatorname{Tr}_{\mathscr{H}_{1}} \rho \in \mathcal{A}_{2}\right\}$ and consider the conjecture

$$
C_{\chi}\left(\Phi_{1} \otimes \Phi_{2} ; \mathcal{A}_{1} \otimes \mathcal{A}_{2}\right)=C_{\chi}\left(\Phi_{1} ; \mathcal{A}_{1}\right)+C_{\chi}\left(\Phi_{2} ; \mathcal{A}_{2}\right),
$$

which apparently implies (6).

Theorem 5.3. Let $\Phi_{1}$ and $\Phi_{2}$ be two fixed channels. The following properties are equivalent:

(i) Equality (34) holds for arbitrary linear constraints $\mathcal{A}_{1}, \mathcal{A}_{2}$.

(ii) Equality (34) holds for arbitrary closed $\mathcal{A}_{1}, \mathcal{A}_{2}$.

(iii) For arbitrary $\rho_{12} \in \mathfrak{S}\left(\mathscr{H}_{1} \otimes \mathscr{H}_{2}\right)$,

$$
\chi_{\Phi_{1} \otimes \Phi_{2}}\left(\rho_{12}\right) \leq \chi_{\Phi_{1}}\left(\rho_{1}\right)+\chi_{\Phi_{2}}\left(\rho_{2}\right) .
$$

(iv) Inequality (31) holds for arbitrary $\rho_{12} \in \mathfrak{S}\left(\mathcal{H}_{1} \otimes \mathscr{H}_{2}\right)$.

Here each property is easily seen to imply the preceding one, while the implication (i) $\Rightarrow$ (iv) is nontrivial. By Proposition 5.1 any of these properties imply the additivity properties (12), (6).

5.3. The convex duality formulation. In [4], tools from convex analysis were applied to study the relation of the additivity problem to superadditivity of entanglement 
of formation. Here we apply a similar approach to the conjecture (31). Given a channel $\Phi$, its output entropy $H(\Phi(\rho))$ is a continuous concave function on the state space $\mathfrak{S}(\mathcal{H})$. Consider its modified Legendre transform

$$
\begin{aligned}
H_{\Phi}^{*}(X) & =\min _{\rho \in \mathfrak{S}(\mathcal{H})}\{\operatorname{Tr} \rho X+H(\Phi(\rho))\} \\
& =\min _{\rho \in \mathfrak{S}(\mathscr{H})}\left\{\operatorname{Tr} \rho X+\hat{H}_{\Phi}(\rho)\right\}, \quad X \in \mathfrak{M}_{h}(\mathcal{H}),
\end{aligned}
$$

where $\mathfrak{M}_{h}(\mathscr{H})$ is a real normed space of Hermitian operators in $\mathscr{H}$.

Now let $\Phi_{1}, \Phi_{2}$ be two channels.

Lemma 5.4. The superadditivity (31) of the convex closure $\hat{H}_{\Phi}(\rho)$ is equivalent to the following additivity property of $H_{\Phi}^{*}(X)$ :

$$
H_{\Phi_{1} \otimes \Phi_{2}}^{*}\left(X_{1} \otimes I_{2}+I_{1} \otimes X_{2}\right)=H_{\Phi_{1}}^{*}\left(X_{1}\right)+H_{\Phi_{2}}^{*}\left(X_{2}\right),
$$

for all $X_{1} \in \mathfrak{M}_{h}\left(\mathscr{H}_{1}\right), X_{2} \in \mathfrak{M}_{h}\left(\mathscr{H}_{2}\right)$.

Since $H_{\Phi}^{*}(0)=\check{H}(\Phi)$, by letting $X_{1}=X_{2}=0$, the relation (37) implies additivity of the minimal output entropy (12).

5.4. The global equivalence. A remarkable result was obtained by Shor [48] who showed that different forms of the additivity conjecture become equivalent if one considers their validity for all channels. Here we describe a basic construction from [48] which in combination with Proposition 5.1 and Theorem 5.3 suffices for the proof of the following result.

Theorem 5.5. The conjectures (6), (12), (31), (34) are globally equivalent in the sense that if one of them holds true for all channels $\Phi_{1}, \Phi_{2}$, then any of the others is also true for all channels.

Let us argue that if additivity of the minimal output entropy (12) holds for all channels, then (37) holds for all channels. By Lemma 5.4 this will imply (31) and hence, by Theorem 5.3, all the other properties.

First of all we observe that $H_{\Phi}^{*}(X+\lambda I)=H_{\Phi}^{*}(X)+\lambda$, which implies that it is sufficient to establish (37) only for $X_{1}, X_{2} \geq 0$. The idea of proof is to build, for any channel $\Phi$ and $X \geq 0$, a sequence of channels $\Phi_{X, n}^{\prime}$ such that

$$
\min _{\rho} H\left(\Phi_{X, n}^{\prime}(\rho)\right) \equiv \check{H}\left(\Phi_{X, n}^{\prime}\right)=\min _{\rho}[H(\Phi(\rho))+\operatorname{Tr} \rho X]+o(1) \equiv H_{\Phi}^{*}(X)+o(1) .
$$

One can then apply the convex duality argument to deduce for the original channels the additivity property (37), which is equivalent to (31), from the additivity of the minimal output entropy for channels $\Phi_{X, n}^{\prime}$.

Given a channel $\Phi: \mathfrak{M}(\mathcal{H}) \rightarrow \mathfrak{M}\left(\mathscr{H}^{\prime}\right)$ and a positive $X \in \mathcal{H}$, the new channel $\Phi_{X, n}^{\prime}$ is constructed as follows. Choose a constant $c \geq\|X\|$, then $E=c^{-1} X$ satisfies $0 \leq E \leq I$. Let $q_{n} \in(0,1)$ be such that

$$
\left(1-q_{n}\right) \log n=c, \quad n=2,3, \ldots
$$


Then $\Phi_{X, n}^{\prime}: \mathfrak{M}(\mathscr{H}) \rightarrow \mathfrak{M}\left(\mathscr{H}_{n}^{\prime}\right)$, where $\mathscr{H}_{n}^{\prime}=\mathscr{H}^{\prime} \oplus \mathscr{H}_{n} \oplus \mathbb{C}$, acts on $\rho \in \mathfrak{M}(\mathscr{H})$ as follows:

$$
\Phi_{X, n}^{\prime}(\rho)=\left[\begin{array}{ccc}
q_{n} \Phi(\rho) & 0 & 0 \\
0 & \left(1-q_{n}\right)(\operatorname{Tr} \rho E) \frac{I_{n}}{n} & 0 \\
0 & 0 & \left(1-q_{n}\right) \operatorname{Tr} \rho(I-E)
\end{array}\right] .
$$

This is an orthogonal convex sum of CP maps, preserving trace, and hence is a channel.

The intuition is that the action of $\Phi_{X, n}^{\prime}(\rho)$ can be described as follows. With probability $q_{n}$ (which tends to 1 as $n \rightarrow \infty$ ) it acts as the channel $\Phi$, resulting in the state $\Phi(\rho)$. With probability $\left(1-q_{n}\right)$, however, a quantum measurement described by the resolution of the identity (the quantum observable) $\{E, I-E\}$ is made, so that the first outcome appears with probability $\operatorname{Tr} \rho E$, while the second appears with probability $\operatorname{Tr} \rho(I-E)$. In the first case the output is the chaotic state $\frac{I_{n}}{n}$ in the $n$-dimensional unitary space $\mathscr{H}_{n}$; in the second case the output is a pure state orthogonal to $\mathscr{H}^{\prime} \oplus \mathscr{H}_{n}$. In this way the channel $\Phi_{X, n}^{\prime}(\rho)$ with high probability $q_{n}$ acts as the initial channel, while with a small probability $\left(1-q_{n}\right)(\operatorname{Tr} \rho E)$ outputs a high dimensional chaotic state $\frac{I_{n}}{n}$, providing the knowledge about the value of $\operatorname{Tr} \rho E=c^{-1} \operatorname{Tr} \rho X$ involved in the definition of $H_{\Phi}^{*}(X)$. This is formalized by proving the uniform estimate

$$
H\left(\left(\Phi_{X, n}^{\prime} \otimes \Phi_{2}\right)\left(\rho_{12}\right)\right)=q_{n} H\left(\left(\Phi \otimes \Phi_{2}\right)\left(\rho_{12}\right)\right)+\operatorname{Tr} \rho_{1} X+o(1),
$$

double application of which reduces the property (37) for initial channels to the additivity of the minimal output entropy for the channels $\Phi_{X, n}^{\prime}$.

A modification of this construction can be also used to show that if the unconstrained additivity (6) holds for all channels, then additivity (34) for all channels with arbitrary constraints holds as well [26]. This completes the global equivalence.

\section{Infinite-dimensional channels}

We have seen that the additivity problem is not completely solved even for the minimal dimension 2. Nevertheless there are several good reasons to consider the problem in infinite dimensions.

There is an important and interesting class of Bosonic Gaussian channels, see [28], which act in infinite dimensional Hilbert space. Analysis of continuity properties of the entropic characteristics of an infinite-dimensional channel becomes important since, as is well known, the entropy may then have a rather pathological behavior. It is only lower semicontinuous and "almost everywhere" infinite in the infinitedimensional case [52]. Another issue is the study of conditions for compactness of subsets of quantum states and ensembles, giving a key for attainability of extrema in expressions for the capacity and the convex closure of the output entropy.

The proof of global equivalence of different forms of the additivity conjecture for finite dimensional channels (Section 5.4), using infinitely growing channel extensions 
in fact relies upon the discontinuity of the $\chi$-capacity as a function of the channel in infinite dimensions. This also calls for a study of continuity properties of the entropic quantities related to the classical capacity of infinite dimensional channels. Such a study was undertaken in a series of works [23], [27], [46]. In particular it was shown that in spite of the aforementioned discontinuities, additivity for all finite-dimensional channels implies additivity of the $\chi$-capacity of infinite-dimensional channels with arbitrary constraints [46].

There are two important features essential for channels in infinite dimensions. One is the necessity of the input constraints (such as mean energy constraint for Gaussian channels) to prevent from infinite capacities (although considering input constraints was shown quite useful also in the study of the additivity conjecture for channels in finite dimensions [26]). The other is the natural appearance of infinite, and, in general, "continuous" state ensembles understood as probability measures on the set of all quantum states. By using compactness criteria from probability and operator theory one can show that the set of all such generalized ensembles with the barycenter in a compact set of states is itself weakly compact. With this in hand a sufficient condition for existence of an optimal generalized ensemble for a constrained quantum channel can be given. This condition can be efficiently verified in the case of Bosonic Gaussian channels with constrained mean energy [27].

However apart from mere existence one would like to have an explicit description of the optimal states and ensembles in the case of quantum Gaussian channels. In classical information theory Gaussian channels have Gaussian maximizers, and there is an analytical counterpart of this phenomenon for $(q \rightarrow p)$-norms of integral operators with Gaussian kernels, see [39]. Whether a similar description holds true for Bosonic Gaussian channels is another open question (for some partial results in this direction see [28], [17], [18], [55]). We only mention here that a positive solution of this question may also depend on the validity of the multiplicativity/ additivity conjecture [39], [55].

\section{References}

[1] Alicki, R., Fannes, M., Note on multiple additivity of minimal Rényi entropy output of the Werner-Holevo channels. quant-ph/0407033.

[2] Amosov, G. G., Holevo, A. S., On the multiplicativity conjecture for quantum channels. Theor. Probab. Appl. 47 (1) (2002), 143-146.

[3] Amosov, G. G., Holevo, A. S., and Werner, R. F., On some additivity problems in quantum information theory. Probl. Inform. Transm. 36 (4) (2000), 25-34.

[4] Audenaert, K. M. R., Braunstein, S. L., On strong superadditivity of the entanglement of formation. Commun. Math. Phys. 246 (2004), 443-452.

[5] Bennett, C. H., Fuchs, C. A., Smolin, J. A., Entanglement-enhanced classical communication on a noisy quantum channel. In Quantum Communication, Computing and Measurement, Proc. QCM96 (ed. by O. Hirota, A. S. Holevo and C. M. Caves), Plenum, New York 1997, 79-88. 
[6] Bennett, C. H., Shor, P. W., Quantum information theory. IEEE Trans. Inform. Theory 44 (1998), 2724-2742.

[7] Bhatia, R., Matrix Analysis. Grad. Texts in Math. 169, Springer-Verlag, New York 1997.

[8] Carlen, E. A., Lieb, E. H., A Minkowski type trace inequality and strong subadditivity of quantum entropy. In Differential operators and spectral theory (ed. by V. Buslaev, M. Solomyak and D. Yafaev), Amer. Math. Soc. Transl. Ser. 2 189, Amer. Math. Soc., Providence, RI, 1999, 59-68.

[9] Choi, M.-D., Completely positive maps on complex matrices. Linear Algebra Appl. 10 (1975), 285-290.

[10] Cover, T. M., Thomas, J. A., Elements of Information Theory. J. Wiley and Sons, New York 1991.

[11] Datta, N., Holevo, A. S., Complementarity and additivity for depolarizing channels. quant$\mathrm{ph} / 0510145$.

[12] Datta, N., Holevo, A. S., Suhov, Y. M., A quantum channel with additive minimum output entropy. quant-ph/0408176.

[13] Datta, N., Multiplicativity of maximal $p$-norms in Werner-Holevo channels for $1 \leq p \leq 2$. quant-ph/0410063.

[14] Devetak, I., Hayden, A. W., Winter, A., A resource framework for quantum Shannon theory. quant-ph/0512015.

[15] Devetak, I., Junge, M., King, C., Ruskai, M. B., Multiplicativity of completely bounded p-norms implies a new additivity result. quant-ph/0506196.

[16] Fukuda, M., Holevo, A. S., On Weyl-covariant channels. quant-ph/0510148.

[17] Giovannetti, V., Lloyd, S., Maccone, L., Shapiro, J. H., Yen, B. J., Minimum Rényi and Wehrl entropies at the output of bosonic channels. quant-ph/0404037.

[18] Giovannetti, V., Lloyd, S., Additivity properties of a Gaussian channel. quant-ph/0403075.

[19] Holevo, A. S., The capacity of quantum communication channel with general signal states. IEEE Trans. Inform. Theory 44 (1) (1998), 269-272.

[20] Holevo, A. S., Quantum coding theorems. Russ. Math. Surveys 53 (1998), 1295-1331.

[21] Holevo, A. S., Statistical structure of quantum theory. Lect. Notes Phys. Monogr. 67, Springer-Verlag, Berlin 2001.

[22] Holevo, A. S., An introduction to quantum information theory. MCCME (Moscow Independent University), Moscow 2002.

[23] Holevo, A. S., Classical capacities of constrained quantum channel. Theory Probab. Appl. 48 (2003), 359-374.

[24] Holevo, A. S., Additivity conjecture and covariant channels. Int. J. Quant. Inform. 3 (1) (2005), 41-48.

[25] Holevo, A. S., On complementary channels and the additivity problem. quant-ph/0509101.

[26] Holevo, A. S., Shirokov, M. E., On Shor's channel extension and constrained channels. Commun. Math. Phys. 249 (2004), 417-430.

[27] Holevo, A. S., Shirokov, M. E., Continuous ensembles and the $\chi$-capacity of infinitedimensional channels. quant-ph/0403072.

[28] Holevo, A. S., Werner, R. F., Evaluating capacities of Bosonic Gaussian channels. Phys. Rev. A. 63 (2001), 032312. 
[29] Horodecki, M., Shor, P. W., Ruskai, M. B., General entanglement breaking channels. Rev. Math. Phys. 15 (2003), 629-641.

[30] Magaril-Il'yaev, G. G., Tikhomirov, B. M., Convex analysis: theory and applications. Editorial URSS, Moscow 2000, Transl. Math. Monogr. 222, Amer. Math. Soc, Providence, RI, 2003.

[31] King, C., An application of the matrix inequality in quantum information theory. quant$\mathrm{ph} / 0412046$.

[32] King, C., Maximal p-norms of entanglement breaking channels. quant-ph/0212057.

[33] King, C., Additivity for a class of unital qubit channels. quant-ph/0103156.

[34] King, C., The capacity of the quantum depolarizing channel. quant-ph/0204172.

[35] King, C., Koldan, N., New multiplicativity results for qubit maps. quant-ph/0512185.

[36] King, C., Matsumoto, K., Natanson, M., and Ruskai, M. B., Properties of conjugate channels with applications to additivity and multiplicativity. quant-ph/0509126.

[37] King, C., Nathanson, M., Ruskai, M.-B., Multiplicativity properties of entrywise positive maps on matrix algebras. quant-ph/0409181.

[38] King, C., Ruskai, M.-B., Comments on multiplicativity of maximal $p$-norms when $p=2$. Quantum Inf. Comput. 4 (2004), 500-512.

[39] Lieb, E. H., Gaussian kernels have only Gaussian maximizers, Invent. Math. 102 (1990), 179-208.

[40] Lieb, E. H., Thirring, W. E., Inequalities for the moments of the eigenvalues of the Schrödinger Hamiltonian and their relation to Sobolev inequalities. In Essays in Honor of Valentine Bargmann (ed. by E. H. Lieb, B. Simon, A. Wightman), Stud. Math.Phys., Princeton University Press, 1976, 269-297.

[41] Matsumoto, K., Yura, F., Entanglement cost of antisymmetric states and additivity of capacity of some channels. quant-ph/0306009.

[42] Nielsen, M. A., Chuang, I., Quantum Computation and Quantum Information. Cambridge University Press, Cambridge 2000.

[43] Paulsen, V., Completely bounded maps and operator algebras. Cambridge University Press, Cambridge 2002.

[44] Pisier, G., Non-Commutative Vector Valued $L_{p}$-spaces and Completely p-summing Maps. Astérisque 247 (1998).

[45] Ruskai, M. B., Szarek, S., Werner, E., An analysis of completely-positive trace-preserving maps on $2 \times 2$ matrices. Linear Algebra Appl. 347 (2002), 159-187.

[46] Shirokov, M. E., The Holevo capacity of infinite dimensional channels. Commun. Math. Phys. 262 (2006), 137-159.

[47] Shor, P. W., Additivity of the classical capacity of entanglement-breaking quantum channels. J. Math. Phys. 43 (2003), 4334-4340.

[48] Shor, P. W., Equivalence of additivity questions in quantum information theory. Commun. Math. Phys. 246 (2004), 453-472.

[49] Schumacher, B., Westmoreland,M. D. , Sending classical information via noisy quantum channel. Phys. Rev. A. 56 (1) (1997), 131-138.

[50] Schumacher, B., Westmoreland, M. D. , Optimal signal ensembles. Phys. Rev. A 63 (2001), 022308. 
[51] Stinespring, W. F., Positive functions on $C^{*}$-algebras. Proc. Amer. Math. Soc. 6 (1955), 211-316.

[52] Wehrl, A., General properties of entropy. Rev. Mod. Phys. 50 (1978), 221-250.

[53] Werner, R. F., Holevo, A. S., Counterexample to an additivity conjecture for output purity of quantum channels. J. Math. Phys. 43 (2002), 4353.

[54] Wolf, M. M., Eisert, J., Classical information capacity of a class of quantum channels. quant-ph/0412133.

[55] Wolf, M. M., Giedke, G., Cirac, J. I., Extremality of Gaussian quantum states. quant$\mathrm{ph} / 0509154$.

Steklov Mathematical Institute, Department of Probability Theory and Mathematical Statistics, Gubkina 8, 119991 Moscow, Russian Federation

E-mail: holevo@mi.ras.ru 